Article

\title{
Synthesis and Antiviral Activity of $N$-Phenylbenzamide Derivatives, a Novel Class of Enterovirus 71 Inhibitors
}

\author{
Xing-Yue $\mathrm{Ji}^{\dagger}{ }^{\dagger}$, Hui-Qiang Wang ${ }^{\dagger}$, Lan-Hu Hao, Wei-Ying He, Rong-Mei Gao, Yan-Ping Li *, \\ Yu-Huan Li *, Jian-Dong Jiang and Zhuo-Rong Li \\ Institute of Medicinal Biotechnology, Chinese Academy of Medical Science and Peking Union \\ Medical College, Beijing 100050, China \\ $\dagger$ These authors contributed equally to this work. \\ * Authors to whom correspondence should be addressed; E-Mails: liyanping08@hotmail.com (Y.-P.L.); \\ yuhuanlibj@126.com (Y.-H.L.); Tel.: +86-10-6316-5267 (Y.-P.L.); +86-10-6301-0984 (Y.-H.L.); \\ Fax: +86-10-6301-7302 (Y.-P.L. \& Y.-H.L.).
}

Received: 6 January 2013; in revised form: 14 March 2013 / Accepted: 18 March 2013 /

Published: 21 March 2013

\begin{abstract}
A series of novel $N$-phenylbenzamide derivatives were synthesized and their anti-EV 71 activities were assayed in vitro. Among the compounds tested, 3-amino- $N$-(4bromophenyl)-4-methoxybenzamide (1e) was active against the EV 71 strains tested at low micromolar concentrations, with $\mathrm{IC}_{50}$ values ranging from $5.7 \pm 0.8-12 \pm 1.2 \mu \mathrm{M}$, and its cytotoxicity to Vero cells $\left(\mathrm{TC}_{50}=620 \pm 0.0 \mu \mathrm{M}\right)$ was far lower than that of pirodavir $\left(\mathrm{TC}_{50}=31 \pm 2.2 \mu \mathrm{M}\right)$. Based on these results, compound $1 \mathrm{e}$ is a promising lead compound for the development of anti-EV 71 drugs.
\end{abstract}

Keywords: synthesis; EV 71; $N$-phenylbenzamide

\section{Introduction}

Enterovirus 71 (EV 71) is a single-strained and positive sense RNA virus that belongs to the Picornaviridae family. It was first isolated and identified from patients with central nervous system (CNS) diseases in California between 1969 and 1974 [1]. Since then, outbreaks of EV 71 have been reported in several countries, especially in the Asia Pacific region [2-4]. Diseases caused by an EV 71 infection include hand-foot-mouth disease (HFMD), herpangina (HA) and CNS involvement with fatal 
pulmonary edema [5]. EV 71 infections are a global public health problem causing severe clinical illness and even death in young children. However, there are currently no antiviral drugs or vaccines available in the clinic against EV 71, and the majority of treatments are simply supportive symptom management treatments. Consequently, there is an increasing need for the discovery of effective drugs against EV 71. Additionally, due to the high mutation rate of EV 71, several genotypes have been isolated in the clinic [6], and, at times, more than one genotype has been associated with both complicated and uncomplicated diseases [7]. Therefore, pinpointing the active strain has been unsuccessful. Consequently, the development of anti-EV 71 drugs should be mainly focused on the discovery of compounds with activity against several genotypes of EV 71.

Antiviral screening of our privileged structure library was carried out, and compound 2a (Figure 1) exhibited moderate activity against EV 71 virus (strain $\mathrm{H}, \mathrm{C} 2$ genotype) in vitro with an $\mathrm{IC}_{50}$ value of $18 \pm 1.2 \mu \mathrm{M}$. In order to obtain compounds with more potent activity and superior physicochemical profiles, we synthesized a series of $N$-phenylbenzamide derivatives and evaluated their antiviral activity against four strains (SZ-98, JS-52-3, H, BrCr) of EV 71 in Vero cells. The structure modification was mainly made to the substituents at the C-3 position of the benzene ring A to investigate the importance of amide group for the anti-EV 71 activity. Meanwhile, because there was no substituent on the ring $\mathrm{B}$ of compound $\mathbf{2 a}$, which possibly made it vulnerable to metabolism, we also synthesized derivatives with substituents on the ring B to make them metabolically stable, and also to explore the structure-activity relationships on the ring B. This is the first report of the anti-EV 71 activity of the $N$-phenylbenzamide scaffold, to the best of our knowledge.

Figure 1. The structure of compound 2a.

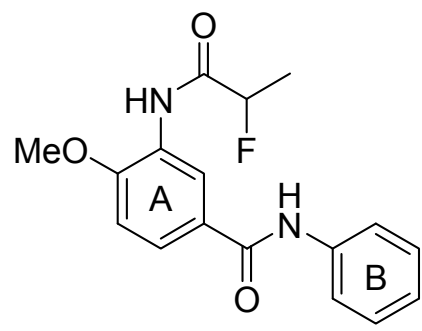

$2 a$

\section{Results and Discussion}

\subsection{Chemistry}

As shown in Schemes 1 and 2, a series of $N$-phenylbenzamide derivatives were designed and synthesized according to our previous reported method [8]. In Scheme 1, 3-amino-4-methoxybenzoic acid was chosen as the starting material and was condensed with a variety of amines, using $N, N^{\prime}$-diisopropylcarbodiimide (DIC) as the coupling reagent and $N$-hydroxybenzotriazole (HOBt) as the activating reagent [9], to yield the intermediate compounds 1a-e. Under the same conditions, compounds 1a, b were condensed with 2-fluoropropionic acid, which was obtained by the hydrolysis of methyl 2-fluoropropanoate, to afford the target compounds $\mathbf{2 a}, \mathbf{b}$. Alkylation of the amine groups of compounds 1a, $\mathbf{c}$ and $\mathbf{d}$ was accomplished by a nucleophilic substitution reaction. To avoid cleavage of 
the amide bond during the reaction, a mild base $\left(\mathrm{NaHCO}_{3}\right)$ was employed as the acid-binding agent. When iodomethane (MeI) was used as the alkylating agent in excess, dialkylation products $\mathbf{3} \mathbf{b}$, $\mathbf{d}$ were also obtained in addition to monoalkylated products. Additionally, by a similar method used to synthesize compounds $\mathbf{2 a}$, b, we also synthesized compound $\mathbf{2} \mathbf{c}$ using 3-methoxy-4-aminobenzoic acid as the starting material.

Scheme 1. The synthetic route to compounds $\mathbf{2 a}-\mathbf{c}$ and $\mathbf{3 a}-\mathbf{g}$.<smiles>COc1ccc(C(=O)O)cc1N</smiles>

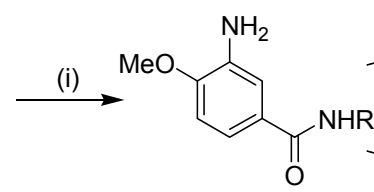

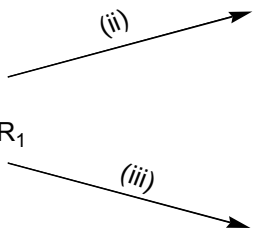

1a: $R_{1}=$ phenyl 1b: $R_{1}=$ cyclohexyl

1c: $R_{1}=4$-chlorophenyl 1d: $R_{1}=3,4,5$-trimethoxyphenyl 1e: $R_{1}=4$-bromophenyl<smiles>COc1ccc(C(=O)Nc2ccccc2)cc1NC(=O)C(C)F</smiles>

2a: $R_{1}=$ phenyl $2 \mathrm{~b}: R_{1}=$ cyclohexyl<smiles>[R7]NC(=O)c1ccc(OC)c(N([R])[R])c1</smiles>

3a: $R_{1}=$ phenyl, $R_{2}=M e, R_{3}=H$ 3b: $R_{1}=4$-chlorophenyl, $R_{2}=R_{3}=M e$ 3c: $\mathrm{R}_{1}=3,4,5$-trimethoxyphenyl, $\mathrm{R}_{2}=\mathrm{Me}, \mathrm{R}_{3}=\mathrm{H}$ 3d: $R_{1}=3,4,5$-trimethoxyphenyl, $R_{2}=R_{3}=M e$ 3e: $R_{1}=$ phenyl, $R_{2}=n-\operatorname{Pr}, R_{3}=H$ 3f: $\mathrm{R}_{1}=4$-chlorophenyl, $\mathrm{R}_{2}=n-\operatorname{Pr}, \mathrm{R}_{3}=\mathrm{H}$ 3g: $\mathrm{R}_{1}=3,4,5$-trimethoxyphenyl, $\mathrm{R}_{2}=n-\mathrm{Pr}, \mathrm{R}_{3}=\mathrm{H}$<smiles>COc1cc(C(=O)O)ccc1N</smiles><smiles>CC#CC</smiles><smiles>COc1cc(C(=O)Nc2ccccc2)ccc1N</smiles><smiles>C#CC</smiles>

$1 f$<smiles>COc1cc(C(=O)Nc2ccccc2)ccc1NC(=O)C(C)F</smiles>

2c

Reagents and conditions: (i) DIC, $\mathrm{HOBt}, \mathrm{R}_{1} \mathrm{NH}_{2}, \mathrm{CH}_{2} \mathrm{Cl}_{2}, \mathrm{rt}, 5 \mathrm{~h}, 60-70 \%$, (ii) 2-fluoropropionic acid, DIC, $\mathrm{HOBt}, \mathrm{CH}_{2} \mathrm{Cl}_{2}$, rt, 5 h, 60-70\%, (iii) MeI/PrI, $\mathrm{NaHCO}_{3}, \mathrm{CH}_{2} \mathrm{Cl}_{2}, \mathrm{rt}, 12 \mathrm{~h}, 30-50 \%$.

Scheme 2. The synthetic route to compounds 5a-f.<smiles>[X]c1cc(C(=O)O)ccc1OC</smiles><smiles>[R]C(=O)[X]c1cc(C(=O)O)ccc1OC</smiles>

4a: $\mathrm{R}_{4}=\mathrm{Et}, \mathrm{X}=\mathrm{NH}$

4b: $R_{4}=E t, X=O$

4c: $R_{4}=M e, X=O$ (ii)<smiles>[R]C(=O)[X]c1cc(C(=O)N([R])c2ccc([R5])cc2)ccc1OC</smiles>

5a: $\mathrm{R}_{4}=\mathrm{Et}, \mathrm{R}_{5}=\mathrm{H}, \mathrm{R}_{6}=\mathrm{H}, \mathrm{X}=\mathrm{NH}$ 5b: $R_{4}=E t, R_{5}=\mathrm{Cl}, \mathrm{R}_{6}=\mathrm{H}, \mathrm{X}=\mathrm{NH}$ 5c: $R_{4}=E t, R_{5}=B r, R_{6}=H, X=N H$ 5d: $R_{4}=\mathrm{Et}, \mathrm{R}_{5}=\mathrm{Cl}, \mathrm{R}_{6}=\mathrm{Me}, \mathrm{X}=\mathrm{O}$ 5e: $R_{4}=M e, R_{5}=C l, R_{6}=H, X=O$ 5f: $R_{4}=M e, R_{5}=H, R_{6}=H, X=O$

Reagents and conditions: (i) TEA, $\mathrm{CH}_{2} \mathrm{Cl}_{2}, \mathrm{R}_{4} \mathrm{COCl}, 0{ }^{\circ} \mathrm{C}-\mathrm{rt}, 6 \mathrm{~h}, 70-80 \%$, (ii) DIC, $\mathrm{HOBt}$, amines, $\mathrm{CH}_{2} \mathrm{Cl}_{2}$, rt, $5 \mathrm{~h}, 60-70 \%$, or $\mathrm{SOCl}_{2}$, reflux $1 \mathrm{~h}$, then $\mathrm{CH}_{2} \mathrm{Cl}_{2}$, TEA, amines , $0{ }^{\circ} \mathrm{C}-\mathrm{rt}, 6 \mathrm{~h}, 60-70 \%$. 
As depicted in Scheme 2, we also synthesized several derivatives with propionyloxy (acetoxy) and propionylamino substituents on the benzene ring A. 3-Hydroxyl/amino-4-methoxybenzoic acid was first reacted in dichloromethane with propionyl chloride or acetyl chloride using triethylamine (TEA) as the base to afford compounds $\mathbf{4 a - c}$. By using a similar method to that used for compounds 1a-e, compounds 5a-f were thus obtained. Alternatively, compounds $\mathbf{4 a}-\mathbf{c}$ were first reacted with thionyl chloride under refluxing conditions to give intermediate chloride products, which were condensed with a substituted arylamine to yield the target compounds $\mathbf{5 a}-\mathbf{f}$. Ultimately, compounds $\mathbf{5 a}-\mathbf{f}$ were obtained in overall yields of $40-60 \%$.

\subsection{Anti-EV 71 Activity}

The EV 71 was divided into three genotypes $\mathrm{A}, \mathrm{B}$ and $\mathrm{C}$ based on nucleotide sequence comparisons, and genotypes $\mathrm{B}$ and $\mathrm{C}$ were subdivided into B1-B5 and C1-C5 [6,10]. Genotype $\mathrm{C}$ is the most common genotype found in mainland China, especially the $\mathrm{C} 4$ subgenotype [4,11]. Consequently, the intermediate compounds 1a, $1 \mathbf{c}$ and 1e, and the final products $2 \mathbf{a}-\mathbf{c}, \mathbf{3 a}-\mathbf{g}$, and $5 \mathbf{a}-\mathbf{f}$ were evaluated for their anti-EV 71 activity in Vero cells against genotypes C4 (strains SZ-98 and JS-52-3), C2 (strain H) and A (strain BrCr). Pirodavir (Figure 2) is a rather potent and broad-spectrum picornavirus inhibitor. It was reported to be effective against $80 \%$ of 16 enteroviruses tested at the concentration of $1.3 \mu \mathrm{g} / \mathrm{mL}$ [12]. Therefore, we employed pirodavir as positive control to validate our model. The $\mathrm{IC}_{50}$ values were calculated by the Reed and Muench method, and the cytotoxicity was monitored by the CPE method.

Figure 2. The chemical structure of pirodavir.

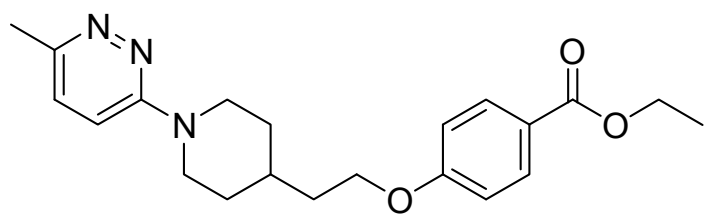

\section{Pirodavir}

As indicated in Table 1, the cytotoxicity of all the synthesized compounds was far lower than that of pirodavir, and most of the synthesized $\mathrm{N}$-phenylamide derivatives exhibited some activity against several strains of EV 71 simultaneously, especially compound 1e, which was active against all the strains tested at low micromolar concentrations $(5.7 \pm 0.80-12 \pm 1.2 \mu \mathrm{M})$. In addition to compound 1e, other compounds also showed moderate levels of inhibitory activity with $\mathrm{IC}_{50}$ values of approximately $15 \mu \mathrm{M}$ including, compound 1c against strain SZ-98, compound $\mathbf{3 g}$ against strains $\mathrm{H}$ and $\mathrm{BrCr}, \mathbf{3 e}$ against strain H, 5c against strains SZ-98, JS-52-3 and H, and compound 5e against strain SZ-98. Moreover, the selectivity index (SI) values of compounds 1c (10-36), 1e (51-110), 3g (16-35), 5b $(>16->27), 5 \mathbf{c}(16-63)$ and 5e (15-37) were also comparable or superior to those of pirodavir (25-52). 
Table 1. The activity of the synthesized $N$-phenylbenzamide derivatives against several strains of EV 71.

\begin{tabular}{|c|c|c|c|c|c|c|c|c|c|}
\hline \multirow[b]{2}{*}{ Cpds } & \multirow[b]{2}{*}{$\begin{array}{l}\mathrm{TC}_{50} \\
(\mu \mathrm{M})\end{array}$} & \multicolumn{2}{|c|}{ SZ-98 } & \multicolumn{2}{|c|}{ JS-52-3 } & \multicolumn{2}{|l|}{ H } & \multicolumn{2}{|c|}{$\mathrm{BrCr}$} \\
\hline & & $\begin{array}{l}\mathrm{IC}_{50} \\
(\mu \mathrm{M})\end{array}$ & SI & $\begin{array}{l}\mathrm{IC}_{50} \\
(\mu \mathrm{M})\end{array}$ & SI & $\begin{array}{l}\mathrm{IC}_{50} \\
(\mu \mathrm{M})\end{array}$ & SI & $\begin{array}{l}\mathrm{IC}_{50} \\
(\mu \mathrm{M})\end{array}$ & SI \\
\hline $1 \mathrm{a}$ & $>820$ & $350 \pm 24$ & 2.3 & $160 \pm 7.9$ & 5.2 & $190 \pm 14$ & 4.3 & $160 \pm 12$ & 5.2 \\
\hline $1 \mathrm{c}$ & $520 \pm 29$ & $15 \pm 0.6$ & 36 & $46 \pm 5.7$ & 12 & $34 \pm 3.6$ & 16 & $56 \pm 4.2$ & 10 \\
\hline $1 \mathrm{e}$ & $620 \pm 0.0$ & $12 \pm 1.1$ & 51 & $9.8 \pm 0.4$ & 64 & $5.7 \pm 0.8$ & 110 & $9.1 \pm 1.4$ & 68 \\
\hline $2 a$ & $630 \pm 0.0$ & $>630$ & - & $160 \pm 5.7$ & $>3.9$ & $18 \pm 1.2$ & $>35$ & $>630$ & - \\
\hline $2 b$ & $>620$ & $>620$ & - & $210 \pm 0.0$ & $>3.0$ & $430 \pm 36$ & $>1.4$ & $360 \pm 26$ & $>1.7$ \\
\hline $2 c$ & $>630$ & $110 \pm 12$ & $>5.8$ & $34 \pm 2.8$ & & $41 \pm 2.9$ & $>16$ & $90 \pm 9.5$ & $>7.0$ \\
\hline $3 a$ & $780 \pm 0.0$ & $>260$ & - & $260 \pm 0.0$ & 3.0 & $260 \pm 0.0$ & 3.0 & $180 \pm 14$ & 4.3 \\
\hline $3 b$ & $510 \pm 38$ & $73 \pm 8.6$ & 7.0 & $42 \pm 3.1$ & 12 & $31 \pm 4.3$ & 16 & $50 \pm 3.8$ & 10 \\
\hline $3 c$ & $280 \pm 5.9$ & $64 \pm 0.0$ & 4.3 & $37 \pm 1.9$ & 7.5 & $37 \pm 1.2$ & 7.5 & $44 \pm 1.0$ & 6.2 \\
\hline 3d & $>560$ & $320 \pm 32$ & $>1.7$ & $>560$ & - & $190 \pm 0.0$ & $>3.0$ & $190 \pm 0.0$ & $>3.0$ \\
\hline $3 e$ & $410 \pm 22$ & $>78$ & - & $34 \pm 4.6$ & 12 & $20 \pm 1.2$ & 20 & $45 \pm 0.0$ & 9.0 \\
\hline $3 f$ & 120 & $>70$ & - & $>70$ & - & $>70$ & - & $>70$ & - \\
\hline $3 g$ & 530 & $>59$ & - & $34 \pm 2.5$ & 16 & $15 \pm 0.0$ & 35 & $20 \pm 3.1$ & 27 \\
\hline $5 a$ & $>670$ & $>75$ & - & $58 \pm 4.9$ & $>12$ & $96 \pm 10$ & $>7.0$ & $45 \pm 10$ & 9.0 \\
\hline $5 b$ & $>600$ & $38 \pm 2.9$ & $>16$ & $39 \pm 1.9$ & $>16$ & $22 \pm 2.9$ & $>27$ & $>67$ & - \\
\hline $5 c$ & $530 \pm 0.0$ & $11 \pm 0.8$ & 47 & $20 \pm 3.1$ & 27 & $8.4 \pm 1.2$ & 63 & $34 \pm 0.0$ & 16 \\
\hline $5 d$ & $280 \pm 21$ & $190 \pm 12$ & 1.4 & $44 \pm 3.7$ & 6.2 & $65 \pm 0.0$ & 4.3 & $53 \pm 2.4$ & 5.2 \\
\hline $5 e$ & $470 \pm 22$ & $13 \pm 2.4$ & 37 & $32 \pm 3.6$ & 14 & $22 \pm 4.7$ & 21 & $32 \pm 3.9$ & 15 \\
\hline $5 f$ & $360 \pm 39$ & $90 \pm 11$ & 4.0 & $98 \pm 4.7$ & 3.7 & $47 \pm 6.1$ & 7.6 & $95 \pm 9.0$ & 3.8 \\
\hline Pirodavir & $31 \pm 2.2$ & $1.2 \pm 0.2$ & 25 & $1.0 \pm 0.2$ & 30 & $0.6 \pm 0.1$ & 52 & $1.0 \pm 0.2$ & 30 \\
\hline
\end{tabular}

$\mathrm{TC}_{50}$ was defined as the concentration that inhibits $50 \%$ cellular growth in comparison with untreated controls and calculated by Reed and Muench method. $\mathrm{IC}_{50}(50 \%$ inhibitory concentration) was calculated by Reed \& Muench method. The selectivity index $(\mathrm{SI})$ was calculated as the ratio of $\mathrm{TC}_{50} / \mathrm{IC}_{50}$. “-" stands for no antiviral selectivity.

In terms of structure-activity relationships, benzene ring B was essential for anti-EV 71 activity. For example, when benzene ring B was replaced by a cyclohexyl group (2b), no anti-EV 71 activity was observed. On the basis of the activity of compounds $\mathbf{1 c}, \mathbf{1 e}, \mathbf{5 b}, \mathbf{5 c}$ and $\mathbf{5 e}$ against EV 71, it was concluded that the introduction of electron-withdrawing groups $(\mathrm{Cl}$ and $\mathrm{Br}$, especially $\mathrm{Br})$ at the para position of benzene ring B increased anti-EV 71 activity. Substitution of acetoxy groups $(\mathbf{5 e}, \mathbf{f})$ for the propionylamino group $(\mathbf{5 a}, \mathbf{b})$ on benzene ring A had little influence on anti-EV 71 activity. Because the activity of compounds with propionyl substituent at the amino group $(\mathbf{5 b}, \mathbf{c})$ was slightly inferior to their unsubstituted counterparts (1c, e), it can be concluded that the propionyl substituent at the amino group disfavors the anti-EV 71 activity. Compound $\mathbf{5 d}$ presented less potent activity against EV 71 in comparison to 5e. Therefore, it is possible that $\mathrm{H}$-bond donor group at the position of $\mathrm{R}_{6}$ substituent favors the anti-EV 71 activity. 


\section{Experimental}

\subsection{General}

All reagents and solvents were purchased from J\&K and Alfa Aesar Chemicals, and were used without purification. ${ }^{1} \mathrm{H}-\mathrm{NMR}$ and ${ }^{13} \mathrm{C}-\mathrm{NMR}$ spectra were recorded in $\mathrm{CDCl}_{3}$ or DMSO- $d_{6}$ on a Varian Inova 400/500 MHz spectrometer (Varian, San Francisco, CA, USA). Chemical shift was reported in parts per million relative to tetramethylsilane as the internal standard. Melting points were determined with a X6 microscope melting point apparatus and were uncorrected. Electrospray ionisation (ESI) high-resolution mass spectra (HRMS) were obtained on an MDS SCIEX Q-Trap mass spectrometer. The area normalization purities of the tested compounds were $>95 \%$ as determined using analytical high-performance liquid chromatography (HPLC method for all compounds: detector wavelength: $260 \mathrm{~nm}$, column temperature: $25^{\circ} \mathrm{C}$, column: ODS $(5 \mu \mathrm{m}, 4.6 \times 250 \mathrm{~mm})$, mobile phase: $\mathrm{methanol} / \mathrm{water}=$ $75 / 25$, flow rate $=1 \mathrm{~mL} / \mathrm{min}$ ). For the synthesis and spectral data of compounds $1 \mathbf{a}, \mathbf{2 a}, \mathbf{4 a}, \mathbf{4 b}, \mathbf{5 a}$ and 5c, please refer to our previous paper [8].

\subsubsection{General Procedure for the Synthesis of Compound $\mathbf{1 b}-\mathbf{f}, \mathbf{5 b}, \mathbf{5 e}, \mathbf{5 f}$}

3-Amino-4-methoxybenzoic acid/4a, c $(1.20 \mathrm{mmol})$ was dissolved in $\mathrm{CH}_{2} \mathrm{Cl}_{2}(20 \mathrm{~mL})$, and DIC $(1.82 \mathrm{mmol})$ and HOBt $(1.82 \mathrm{mmol})$ was added to the solution. The resulting mixture was stirred for $0.5 \mathrm{~h}$ at room temperature, after which amines $(1.68 \mathrm{mmol})$ was added. After approximately $12 \mathrm{~h}$, the reaction was quenched by the addition of $0.5 \mathrm{~N} \mathrm{NaOH}$ solution $(20 \mathrm{~mL})$, and the organic layer was separated and washed with dilute hydrochloric acid $(10 \%, 30 \mathrm{~mL})$ and brine $(30 \mathrm{~mL})$, successively. The solution was then dried over anhydrous $\mathrm{MgSO}_{4}$, filtered and concentrated, and the crude residue was purified over silica gel (petroleum/ethyl acetate $=3 / 2$ ) to give the corresponding compounds.

3-Amino-N-cyclohexyl-4-methoxybenzamide (1b). White solid, yield: $65 \%$. mp: $171-172{ }^{\circ} \mathrm{C} .{ }^{1} \mathrm{H}-\mathrm{NMR}$ $\left(400 \mathrm{MHz}, \mathrm{DMSO}-d_{6}\right) \delta: 1.13(1 \mathrm{H}, \mathrm{m}), 1.24(4 \mathrm{H}, \mathrm{m}), 1.62(1 \mathrm{H}, \mathrm{m}), 1.75(4 \mathrm{H}, \mathrm{m}), 3.63(1 \mathrm{H}, \mathrm{m}), 3.79$ $(3 \mathrm{H}, \mathrm{s}), 4.79(2 \mathrm{H}, \mathrm{br}), 6.80(1 \mathrm{H}, \mathrm{d}, J=8.4 \mathrm{~Hz}), 7.07(1 \mathrm{H}, \mathrm{d}, J=8.4 \mathrm{~Hz}), 7.12(1 \mathrm{H}, \mathrm{s}), 8.25(1 \mathrm{H}, \mathrm{s})$. ESI-HRMS $m / z$ : $249.16018\left(\mathrm{M}+\mathrm{H}^{+}\right)\left(\mathrm{Calcd}\right.$ for $\left.\mathrm{C}_{14} \mathrm{H}_{21} \mathrm{~N}_{2} \mathrm{O}_{2}: 249.16030\right)$.

3-Amino-N-(4-chlorophenyl)-4-methoxybenzamide (1c). White solid, yield: $66 \%$ mp $152-154{ }^{\circ} \mathrm{C}$. ${ }^{1} \mathrm{H}-\mathrm{NMR}\left(400 \mathrm{MHz}, \mathrm{DMSO}-d_{6}\right) \delta: 3.84(\mathrm{~s}, 3 \mathrm{H}), 5.46(2 \mathrm{H}, \mathrm{br}), 6.88(\mathrm{~d}, 1 \mathrm{H}, J=6.8 \mathrm{~Hz}), 7.19(\mathrm{~m}, 2 \mathrm{H})$, $7.37(\mathrm{~d}, 2 \mathrm{H}, J=8.0 \mathrm{~Hz}), 7.78(\mathrm{~d}, 2 \mathrm{H}, J=8.0 \mathrm{~Hz}), 10.07(\mathrm{~s}, 1 \mathrm{H}) .{ }^{13} \mathrm{C}-\mathrm{NMR}\left(100 \mathrm{MHz}, \mathrm{CDCl}_{3}\right) \delta: 55.6$, 109.6, 113.4, 117.4, 121.2, 124.7, 128.9, 129.2, 136.5, 150.1, 156.9. ESI-HRMS m/z: 277.07487 $\left(\mathrm{M}+\mathrm{H}^{+}\right)\left(\right.$Calcd for $\left.\mathrm{C}_{14} \mathrm{H}_{14} \mathrm{ClN}_{2} \mathrm{O}_{2}: 277.07438\right)$.

3-Amino-4-methoxy-N-(3,4,5-trimethoxyphenyl)benzamide (1d). White solid, yield: $65 \%$. mp: 140-142 ${ }^{\circ} \mathrm{C}$. ${ }^{1} \mathrm{H}-\mathrm{NMR}\left(500 \mathrm{MHz}, \mathrm{DMSO}-d_{6}\right) \delta: 3.62(3 \mathrm{H}, \mathrm{s}), 3.76(6 \mathrm{H}, \mathrm{s}), 3.83(3 \mathrm{H}, \mathrm{s}), 4.92(2 \mathrm{H}, \mathrm{s}), 6.88(1 \mathrm{H}, \mathrm{d}$, $J=8.0 \mathrm{~Hz}), 7.22(4 \mathrm{H}, \mathrm{m}), 9.85(1 \mathrm{H}, \mathrm{s})$. ESI-HRMS $m / z: 333.14778\left(\mathrm{M}+\mathrm{H}^{+}\right)\left(\right.$Calcd for $\mathrm{C}_{17} \mathrm{H}_{21} \mathrm{~N}_{2} \mathrm{O}_{5}$ : $333.14505)$.

3-Amino-N-(4-bromophenyl)-4-methoxybenzamide (1e). White solid, yield: $68 \%$. mp 190-192 ${ }^{\circ} \mathrm{C}$. ${ }^{1} \mathrm{H}-\mathrm{NMR}\left(500 \mathrm{MHz}, \mathrm{DMSO}-d_{6}\right) \delta: 3.84(\mathrm{~s}, 3 \mathrm{H}), 4.97$ (s, 2H), $6.90(\mathrm{~d}, 1 \mathrm{H}, J=8.0 \mathrm{~Hz}), 7.21$ (m, 2H), 
$7.51(\mathrm{~d}, 2 \mathrm{H}, J=8.0 \mathrm{~Hz}), 7.74(\mathrm{~d}, 2 \mathrm{H}, J=8.0 \mathrm{~Hz}), 10.10(\mathrm{~s}, 1 \mathrm{H}) .{ }^{13} \mathrm{C}-\mathrm{NMR}\left(100 \mathrm{MHz}, \mathrm{CDCl}_{3}\right) \delta: 55.6$, 109.6, 113.6, 116.6, 117.3, 121.5, 127.3, 131.9, 136.5, 137.3, 150.1, 165.5. ESI-HRMS m/z: 321.02365 $\left(\mathrm{M}+\mathrm{H}^{+}\right)\left(\right.$Calcd for $\left.\mathrm{C}_{14} \mathrm{H}_{14} \mathrm{BrN}_{2} \mathrm{O}_{2}: 321.02387\right)$.

4-Amino-3-methoxy-N-phenylbenzamide (1f). White solid, yield: $70 \%$. mp: $174-176^{\circ} \mathrm{C} .{ }^{1} \mathrm{H}-\mathrm{NMR}(400$ $\left.\mathrm{MHz}, \mathrm{DMSO}-d_{6}\right) \delta: 3.82(\mathrm{~s}, 3 \mathrm{H}), 5.37(2 \mathrm{H}, \mathrm{s}), 6.66(1 \mathrm{H}, \mathrm{d}, J=8.0 \mathrm{~Hz}), 7.03(1 \mathrm{H}, \mathrm{d}, J=8.0 \mathrm{~Hz}), 7.32$ $(2 \mathrm{H}, \mathrm{m}), 7.45(2 \mathrm{H}, \mathrm{m}), 7.73(2 \mathrm{H}, \mathrm{d}, J=7.6 \mathrm{~Hz}), 9.77(1 \mathrm{H}, \mathrm{s})$. ESI-HRMS $m / z: 243.11329\left({\left.\mathrm{M}+\mathrm{H}^{+}\right)}^{+}\right.$ (Calcd for $\mathrm{C}_{14} \mathrm{H}_{15} \mathrm{~N}_{2} \mathrm{O}_{2}: 243.11335$ ).

$\mathrm{N}$-(4-Chlorophenyl)-4-methoxy-3-propionamidobenzamide (5b). White solid, yield: 70\%. mp: 130-132 ${ }^{\circ} \mathrm{C}$. ${ }^{1} \mathrm{H}-\mathrm{NMR}\left(400 \mathrm{MHz}, \mathrm{CDCl}_{3}\right) \delta: 1.07(3 \mathrm{H}, \mathrm{t}, J=7.6 \mathrm{~Hz}), 2.42(2 \mathrm{H}, \mathrm{q}, J=7.6 \mathrm{~Hz}), 3.96(3 \mathrm{H}, \mathrm{s}), 6.98$ $(1 \mathrm{H}, \mathrm{d}, J=8.0 \mathrm{~Hz}), 7.32(1 \mathrm{H}, \mathrm{d}, J=8.0 \mathrm{~Hz}), 7.62(2 \mathrm{H}, \mathrm{d}, J=8.0 \mathrm{~Hz}), 7.79(1 \mathrm{H}, \mathrm{dd}, J=8.0$ and $2.0 \mathrm{~Hz}), 7.82(1 \mathrm{H}, \mathrm{s}), 8.09(1 \mathrm{H}, \mathrm{s}), 8.87(1 \mathrm{H}, \mathrm{s})$. ESI-HRMS m/z: $333.10200\left(\mathrm{M}+\mathrm{H}^{+}\right)($Calcd for $\left.\mathrm{C}_{17} \mathrm{H}_{18} \mathrm{ClN}_{2} \mathrm{O}_{3}: 333.10059\right)$.

$\mathrm{N}$-(4-Chlorophenyl)-4-methoxy-3-acetoxybenzamide (5e). White solid, yield: 58\%. mp: $177-179{ }^{\circ} \mathrm{C}$. ${ }^{1} \mathrm{H}-\mathrm{NMR}\left(400 \mathrm{MHz}, \mathrm{DMSO}-d_{6}\right) \delta: 2.34(3 \mathrm{H}, \mathrm{s}), 3.89(3 \mathrm{H}, \mathrm{s}), 6.73(1 \mathrm{H}, \mathrm{d}, J=8.0 \mathrm{~Hz}), 7.01(2 \mathrm{H}, \mathrm{d}$, $J=8.4 \mathrm{~Hz}), 7.13(1 \mathrm{H}, \mathrm{d}, J=8.0 \mathrm{~Hz}), 7.22(2 \mathrm{H}, \mathrm{d}, J=8.4 \mathrm{~Hz}), 8.30(1 \mathrm{H}, \mathrm{s}), 9.01(1 \mathrm{H}, \mathrm{s})$. ESI-HRMS $\mathrm{m} / \mathrm{z}: 320.06891\left(\mathrm{M}+\mathrm{H}^{+}\right)\left(\right.$Calcd for $\left.\mathrm{C}_{16} \mathrm{H}_{15} \mathrm{ClNO}_{4}: 320.06896\right)$.

4-Methoxy-N-phenyl-3-acetoxybenzamide (5f). White solid, yield: $61 \%$. mp: $153-155{ }^{\circ} \mathrm{C} .{ }^{1} \mathrm{H}-\mathrm{NMR}$ $\left(400 \mathrm{MHz}, \mathrm{DMSO}-d_{6}\right) \delta: 2.29(3 \mathrm{H}, \mathrm{s}), 3.85(3 \mathrm{H}, \mathrm{s}), 7.08(1 \mathrm{H}, \mathrm{t}, J=8.0 \mathrm{~Hz}), 7.26(1 \mathrm{H}, \mathrm{d}, J=8.4 \mathrm{~Hz})$, $7.33(2 \mathrm{H}, \mathrm{t}, J=8.0 \mathrm{~Hz}), 7.73(3 \mathrm{H}, \mathrm{m}), 7.92(\mathrm{~d}, 1 \mathrm{H}, J=8.4 \mathrm{~Hz}), 10.11(1 \mathrm{H}, \mathrm{s})$. ESI-HRMS m/z: 286.10799 $\left(\mathrm{M}+\mathrm{H}^{+}\right)\left(\right.$Calcd for $\left.\mathrm{C}_{16} \mathrm{H}_{16} \mathrm{NO}_{4}: 286.10793\right)$.

\subsubsection{General Procedure for the Synthesis of Compounds $\mathbf{2 b}, \mathbf{c}$}

2-Fluoropropanoic acid (2.40 mmol) was dissolved in $\mathrm{CH}_{2} \mathrm{Cl}_{2}(20 \mathrm{~mL})$, and DIC $(3.60 \mathrm{mmol})$ and HOBt $(3.60 \mathrm{mmol})$ was added to the solution. The resulting mixture was stirred for $0.5 \mathrm{~h}$ at room temperature, after which compound $\mathbf{1 b} / \mathbf{1 e}(1.71 \mathrm{mmol})$ was added. After approximately $12 \mathrm{~h}$, the reaction was quenched by the addition of $0.5 \mathrm{~N} \mathrm{NaOH}$ solution $(20 \mathrm{~mL})$, and the organic layer was separated and washed with dilute hydrochloric acid $(10 \%, 30 \mathrm{~mL})$ and brine $(30 \mathrm{~mL})$, successively. The solution was then dried over anhydrous $\mathrm{MgSO}_{4}$, filtered and concentrated, and the crude residue was purified over silica gel (petroleum/ethyl acetate $=4 / 1$ ) to give compounds $\mathbf{2 b}$, $\mathbf{c}$.

N-Cyclohexyl-3-(2-fluoropropanamido)-4-methoxybenzamide (2b). White solid, yield: 73\%. mp: 192-194 ${ }^{\circ} \mathrm{C} .{ }^{1} \mathrm{H}-\mathrm{NMR}\left(400 \mathrm{MHz}, \mathrm{DMSO}-d_{6}\right) \delta: 1.15(1 \mathrm{H}, \mathrm{m}), 1.26(4 \mathrm{H}, \mathrm{m}), 1.55(3 \mathrm{H}, \mathrm{dd}, J=6.4$ and $16.8 \mathrm{~Hz}), 1.61(1 \mathrm{H}, \mathrm{m}), 1.72(4 \mathrm{H}, \mathrm{m}), 3.73(1 \mathrm{H}, \mathrm{m}), 3.93(3 \mathrm{H}, \mathrm{s}), 5.34(1 \mathrm{H}, \mathrm{qq}, J=6.4$ and $48.8 \mathrm{~Hz})$, $7.15(1 \mathrm{H}, \mathrm{d}, J=8.0 \mathrm{~Hz}), 8.06(1 \mathrm{H}, \mathrm{d}, J=8.0 \mathrm{~Hz}), 8.33(1 \mathrm{H}, \mathrm{s}), 9.16(1 \mathrm{H}, \mathrm{s}), 13.63(1 \mathrm{H}, \mathrm{s})$. ESI-HRMS $m / z: 323.17695\left(\mathrm{M}+\mathrm{H}^{+}\right)\left(\right.$Calcd for $\left.\mathrm{C}_{17} \mathrm{H}_{24} \mathrm{FN}_{2} \mathrm{O}_{3}: 323.17710\right)$.

4-(2-Fluoropropanamido)-3-methoxy-N-phenylbenzamide (2c). White solid, yield: 59\%. mp: 142-143 ${ }^{\circ} \mathrm{C}$. ${ }^{1} \mathrm{H}-\mathrm{NMR}\left(500 \mathrm{MHz}, \mathrm{DMSO}-d_{6}\right) \delta: 1.55(3 \mathrm{H}, \mathrm{dd}, J=6.5$ and $20.0 \mathrm{~Hz}), 3.96(3 \mathrm{H}, \mathrm{s}), 5.34(1 \mathrm{H}, \mathrm{qq}$, $J=7.0$ and $48.5 \mathrm{~Hz}), 7.09(1 \mathrm{H}, \mathrm{t}, J=8.0 \mathrm{~Hz}), 7.35(2 \mathrm{H}, \mathrm{t}, J=8.0 \mathrm{~Hz}), 7.61(2 \mathrm{H}, \mathrm{m}), 7.74(2 \mathrm{H}, \mathrm{d}$, 
$J=8.0 \mathrm{~Hz}), 8.17(1 \mathrm{H}, \mathrm{d}, J=8.0 \mathrm{~Hz}), 9.22(1 \mathrm{H}, \mathrm{s}), 10.12(1 \mathrm{H}, \mathrm{s})$. ESI-HRMS $m / z: 317.13087\left(\mathrm{M}+\mathrm{H}^{+}\right)$ (Calcd for $\mathrm{C}_{17} \mathrm{H}_{18} \mathrm{FN}_{2} \mathrm{O}_{3}: 317.13015$ ).

\subsubsection{General Procedure for the Synthesis of Compound 3a-g}

Compound 1a/1c/1d (2.2 mmol) was dissolved in $\mathrm{CH}_{2} \mathrm{Cl}_{2}(15 \mathrm{~mL})$, and $\mathrm{NaHCO}_{3}(2.5 \mathrm{mmol})$ and $\mathrm{MeI} / n$-PrI $(22 \mathrm{mmol})$ were added. The resulting mixture was stirred for $12 \mathrm{~h}$ at room temperature. The mixture was filtered, and the filtrate was concentrated in vacuo to give a residue which was purified over silica gel (petroleum/ethyl acetate $=2 / 1$ ) to give the target compounds $\mathbf{3 a}-\mathbf{g}$.

4-Methoxy-3-methylamino-N-phenylbenzamide (3a). White solid, yield: 33\%. mp: $170-172{ }^{\circ} \mathrm{C}$. ${ }^{1} \mathrm{H}-\mathrm{NMR}\left(400 \mathrm{MHz}, \mathrm{DMSO}-d_{6}\right) \delta: 2.77$ (d, 3H, $J=5.2 \mathrm{~Hz}$ ), 3.84 (s, 3H), 5.19 (q, 1H, $\left.J=5.2 \mathrm{~Hz}\right), 6.88$ $(\mathrm{d}, 1 \mathrm{H}, J=8.0 \mathrm{~Hz}), 7.05(\mathrm{~m}, 2 \mathrm{H}), 7.23(\mathrm{~d}, 1 \mathrm{H}, J=8.0 \mathrm{~Hz}), 7.32(\mathrm{t}, 2 \mathrm{H}, J=8.0 \mathrm{~Hz}), 7.74(\mathrm{~d}, 2 \mathrm{H}$, $J=8.0 \mathrm{~Hz}), 9.93(\mathrm{~s}, 1 \mathrm{H}) .{ }^{13} \mathrm{C}-\mathrm{NMR}\left(100 \mathrm{MHz}, \mathrm{CDCl}_{3}\right) \delta: 30.2,55.6,107.8,108.2,115.0,119.9$, 124.1, 128.1, 129.0, 138.3, 139.5, 149.6, 166.1. ESI-HRMS $m / z: 257.12934\left(\mathrm{M}+\mathrm{H}^{+}\right)($Calcd for $\left.\mathrm{C}_{15} \mathrm{H}_{17} \mathrm{~N}_{2} \mathrm{O}_{2}: 257.12900\right)$.

N-(4-Chlorophenyl)-3-dimethylamino-4-methoxybenzamide (3b). White solid, yield: 40\%. mp: 160-162 ${ }^{\circ} \mathrm{C} .{ }^{1} \mathrm{H}-\mathrm{NMR}\left(400 \mathrm{MHz}, \mathrm{DMSO}-d_{6}\right) \delta: 2.77(6 \mathrm{H}, \mathrm{s}), 3.86(3 \mathrm{H}, \mathrm{s}), 7.03(1 \mathrm{H}, \mathrm{d}, J=8.0 \mathrm{~Hz})$, $7.37(2 \mathrm{H}, \mathrm{d}, J=8.0 \mathrm{~Hz}), 7.43(1 \mathrm{H}, \mathrm{s}), 7.60(1 \mathrm{H}, \mathrm{d}, J=8.0 \mathrm{~Hz}), 7.79(2 \mathrm{H}, \mathrm{d}, J=8.0 \mathrm{~Hz}), 10.12(1 \mathrm{H}, \mathrm{s})$. ${ }^{13} \mathrm{C}-\mathrm{NMR}\left(100 \mathrm{MHz}\right.$, DMSO- $\left.d_{6}\right) \delta: 23.8,56.2,111.4,117.7,122.2,127.2,127.4,128.8,138.9,142.3$, 155.1, 157.3, 165.9. ESI-HRMS $m / z$ : $305.10524\left(\mathrm{M}+\mathrm{H}^{+}\right)\left(\mathrm{Calcd}\right.$ for $\left.\mathrm{C}_{16} \mathrm{H}_{18} \mathrm{ClN}_{2} \mathrm{O}_{2}: 305.10568\right)$.

4-Methoxy-3-methylamino- $N$-(3,4,5-trimethoxyphenyl)benzamide (3c). White solid, yield: 30\%. mp: 147-148 ${ }^{\circ} \mathrm{C} .{ }^{1} \mathrm{H}-\mathrm{NMR}\left(400 \mathrm{MHz}, \mathrm{DMSO}-d_{6}\right) \delta: 2.77(3 \mathrm{H}, \mathrm{s}), 3.62(3 \mathrm{H}, \mathrm{s}), 3.75(6 \mathrm{H}, \mathrm{s}), 3.84(3 \mathrm{H}, \mathrm{s})$, $5.21(1 \mathrm{H}, \mathrm{br}), 6.89(1 \mathrm{H}, \mathrm{d}, J=8.0 \mathrm{~Hz}), 7.01(1 \mathrm{H}, \mathrm{s}), 7.24(3 \mathrm{H}, \mathrm{m}), 9.84(1 \mathrm{H}, \mathrm{s}) .{ }^{13} \mathrm{C}-\mathrm{NMR}(100 \mathrm{MHz}$, $\left.\mathrm{CDCl}_{3}\right) \delta:$ 30.2, 55.6, 56.1, 60.9, 65.8, 97.6, 107.8, 108.6, 114.9, 127.9, 134.4, 134.5, 139.5, 149.6, 153.3, 166.1. ESI-HRMS $m / z$ : $347.16079\left(\mathrm{M}+\mathrm{H}^{+}\right)\left(\right.$Calcd for $\mathrm{C}_{18} \mathrm{H}_{23} \mathrm{~N}_{2} \mathrm{O}_{5}$ : 347.16070).

3-Dimethylamino-4-methoxy-N-(3,4,5-trimethoxyphenyl)benzamide (3d). White solid, yield: 21\%. mp: 120-122 ${ }^{\circ} \mathrm{C} .{ }^{1} \mathrm{H}-\mathrm{NMR}\left(400 \mathrm{MHz}, \mathrm{DMSO}-d_{6}\right) \delta: 2.76(6 \mathrm{H}, \mathrm{s}), 3.62(3 \mathrm{H}, \mathrm{s}), 3.75(6 \mathrm{H}, \mathrm{s}), 3.87$ (3H, s), $7.08(1 \mathrm{H}, \mathrm{d}, J=8.0 \mathrm{~Hz}), 7.20(2 \mathrm{H}, \mathrm{s}), 7.48(1 \mathrm{H}, \mathrm{s}), 7.82(1 \mathrm{H}, \mathrm{d}, J=8.0 \mathrm{~Hz}), 9.98(1 \mathrm{H}, \mathrm{s}) .{ }^{13} \mathrm{C}-\mathrm{NMR}$ $\left(100 \mathrm{MHz}, \mathrm{CDCl}_{3}\right) \delta: 30.2,55.6,56.1,56.1,60.9,97.9,107.8,108.4,110.2,115.2,116.4,124.8,127.2$, 127.8, 134.2, 153.2, 166.3. ESI-HRMS $m / z: 361.17621\left(\mathrm{M}+\mathrm{H}^{+}\right)\left(\right.$Calcd for $\left.\mathrm{C}_{19} \mathrm{H}_{25} \mathrm{~N}_{2} \mathrm{O}_{5}: 361.17635\right)$.

4-Methoxy-N-phenyl-3-(propylamino)benzamide (3e). White solid, yield: 46\%. mp: 121-123 ${ }^{\circ} \mathrm{C}$. ${ }^{1} \mathrm{H}-\mathrm{NMR}\left(400 \mathrm{MHz}, \mathrm{DMSO}-d_{6}\right) \delta: 0.94(3 \mathrm{H}, \mathrm{t}, J=7.2 \mathrm{~Hz}), 1.62(2 \mathrm{H}, \mathrm{m}), 3.09(2 \mathrm{H}, \mathrm{m}), 3.84(3 \mathrm{H}, \mathrm{s})$, $4.93(1 \mathrm{H}, \mathrm{t}, J=5.6 \mathrm{~Hz}), 6.88(1 \mathrm{H}, \mathrm{d}, J=8.4 \mathrm{~Hz}), 7.05(2 \mathrm{H}, \mathrm{m}), 7.23(1 \mathrm{H}, \mathrm{d}, J=8.4 \mathrm{~Hz}), 7.31(2 \mathrm{H}, \mathrm{t}$, $J=8.0 \mathrm{~Hz}), 7.73(2 \mathrm{H}, \mathrm{d}, J=8.0 \mathrm{~Hz}), 9.93(1 \mathrm{H}, \mathrm{s}) .{ }^{13} \mathrm{C}-\mathrm{NMR}\left(100 \mathrm{MHz}, \mathrm{DMSO}-d_{6}\right) \delta: 11.6,21.7,40.1$, 55.6, 107.8, 108.6, 115.6, 120.3, 123.2, 127.4, 128.5, 137.8, 139.4, 148.9, 165.7. ESI-HRMS m/z: $285.15975\left(\mathrm{M}+\mathrm{H}^{+}\right)\left(\right.$Calcd for $\left.\mathrm{C}_{17} \mathrm{H}_{21} \mathrm{~N}_{2} \mathrm{O}_{2}: 285.16006\right)$.

$\mathrm{N}$-(4-Chlorophenyl)-4-methoxy-3-propylaminobenzamide (3f). White solid, yield: 37\%. mp: $168-171{ }^{\circ} \mathrm{C}$. ${ }^{1} \mathrm{H}-\mathrm{NMR}\left(500 \mathrm{MHz}, \mathrm{DMSO}-d_{6}\right) \delta: 0.93(3 \mathrm{H}, \mathrm{t}, J=7.0 \mathrm{~Hz}), 1.59$ (2H, m), 3.09 (2H, m), 3.84 (3H, s), 
$4.93(1 \mathrm{H}, \mathrm{t}, J=5.0 \mathrm{~Hz}), 6.89(1 \mathrm{H}, \mathrm{d}, J=8.0 \mathrm{~Hz}), 7.05(1 \mathrm{H}, \mathrm{s}), 7.24(1 \mathrm{H}, \mathrm{dd}, J=1.5$ and $8.0 \mathrm{~Hz}), 7.36$ $(2 \mathrm{H}, \mathrm{d}, J=8.0 \mathrm{~Hz}), 7.79(2 \mathrm{H}, \mathrm{d}, J=8.0 \mathrm{~Hz}), 10.05(1 \mathrm{H}, \mathrm{s}) .{ }^{13} \mathrm{C}-\mathrm{NMR}\left(100 \mathrm{MHz}, \mathrm{DMSO}-d_{6}\right) \delta: 11.6$, 21.7, 39.9, 55.6, 107.8, 108.7, 115.7, 121.8, 126.8, 127.2, 128.4, 137.9, 138.5, 149.0, 165.9. ESI-HRMS m/z: $319.12139\left(\mathrm{M}+\mathrm{H}^{+}\right)\left(\right.$Calcd for $\left.\mathrm{C}_{17} \mathrm{H}_{20} \mathrm{ClN}_{2} \mathrm{O}_{2}: 319.12133\right)$.

4-Methoxy-3-(propylamino)-N-(3,4,5-trimethoxyphenyl)benzamide (3g). White solid, yield: 41\%. mp: 164-167 ${ }^{\circ} \mathrm{C} .{ }^{1} \mathrm{H}-\mathrm{NMR}\left(500 \mathrm{MHz}, \mathrm{DMSO}-d_{6}\right) \delta: 0.92(3 \mathrm{H}, \mathrm{t}, J=7.0 \mathrm{~Hz}), 1.60(2 \mathrm{H}, \mathrm{m}), 3.09(2 \mathrm{H}, \mathrm{m})$, $3.62(3 \mathrm{H}, \mathrm{s}), 3.75(6 \mathrm{H}, \mathrm{s}), 3.84(3 \mathrm{H}, \mathrm{s}), 4.93(1 \mathrm{H}, \mathrm{br}), 6.89(1 \mathrm{H}, \mathrm{d}, J=8.0 \mathrm{~Hz}), 7.06(1 \mathrm{H}, \mathrm{s}), 7.23(3 \mathrm{H}$, m), $9.84(1 \mathrm{H}, \mathrm{s}) .{ }^{13} \mathrm{C}-\mathrm{NMR}\left(100 \mathrm{MHz}, \mathrm{CDCl}_{3}\right) \delta: 11.5,22.5,45.2,55.5,56.0,60.9,97.6,108.2,108.3$, 114.6, 127.8, 134.5, 134.5, 138.7, 149.4, 153.3, 166.1. ESI-HRMS m/z: $375.19227\left(\mathrm{M}+\mathrm{H}^{+}\right)(\mathrm{Calcd}$ for $\left.\mathrm{C}_{20} \mathrm{H}_{27} \mathrm{~N}_{2} \mathrm{O}_{5}: 375.19200\right)$.

3-Acetoxy-4-methoxybenzoic acid (4c). To a solution of 3-hydroxy-4-methoxybenzoic acid (5.0 g, $30 \mathrm{mmol})$ and $\mathrm{Et}_{3} \mathrm{~N}(4.5 \mathrm{~g}, 45 \mathrm{mmol})$ in $\mathrm{CH}_{2} \mathrm{Cl}_{2}(100 \mathrm{~mL})$ was added acetyl chloride $(3.5 \mathrm{~g}, 45 \mathrm{mmol})$ at $0{ }^{\circ} \mathrm{C}$, and the resulting mixture was warmed to room temperature and then stirred for another $2 \mathrm{~h}$. The reaction mixture was then washed with water $(100 \mathrm{~mL})$, and brine $(100 \mathrm{~mL})$ successively. The organic layer was dried over anhydrous $\mathrm{MgSO}_{4}$, filtered and concentrated, and the crude residue was purified over silica gel (petroleum/ethyl acetate $=4 / 1)$ to give compounds $4 \mathbf{c}$ ( $3.8 \mathrm{~g}$, yield: $60 \%$ ), white solid, mp: $230-231{ }^{\circ} \mathrm{C} .{ }^{1} \mathrm{H}-\mathrm{NMR}\left(400 \mathrm{MHz}, \mathrm{DMSO}-d_{6}\right) \delta: 2.23(3 \mathrm{H}, \mathrm{s}), 3.85(3 \mathrm{H}, \mathrm{s}), 7.35(1 \mathrm{H}, \mathrm{d}$, $J=8.0 \mathrm{~Hz}), 7.83(1 \mathrm{H}, \mathrm{s}), 7.90(1 \mathrm{H}, \mathrm{d}, J=8.0 \mathrm{~Hz}), 12.21(1 \mathrm{H}, \mathrm{s})$. ESI-HRMS $m / z: 209.04513\left(\mathrm{M}^{-} \mathrm{H}^{-}\right)$ (Calcd for $\mathrm{C}_{10} \mathrm{H}_{9} \mathrm{O}_{5}: 209.04500$ ).

$\mathrm{N}$-(4-Chlorophenyl)-N-methyl-4-methoxy-3-propionyloxybenzamide (5d). A mixture of compound $\mathbf{4 b}$ $(0.5 \mathrm{~g}, 2.23 \mathrm{mmol})$ and $\mathrm{SOCl}_{2}(15 \mathrm{~mL}, 206 \mathrm{mmol})$ was heated under reflux for $1 \mathrm{~h}$. The excess $\mathrm{SOCl}_{2}$ was evaporated and the corresponding chloride product was obtained as yellow oil, which was dissolved in $\mathrm{CH}_{2} \mathrm{Cl}_{2}(5 \mathrm{~mL})$ and used without further purification. $N$-methyl-4-chlorobenzenamine $(0.38 \mathrm{~g}, 2.68 \mathrm{mmol})$ was dissolved in $\mathrm{CH}_{2} \mathrm{Cl}_{2}(20 \mathrm{~mL})$, and $\mathrm{Et}_{3} \mathrm{~N}(0.34 \mathrm{~g}, 3.34 \mathrm{mmol})$ was added, and the mixture was cooled to $0{ }^{\circ} \mathrm{C}$ (ice bath). A solution of the acid chloride product in $\mathrm{CH}_{2} \mathrm{Cl}_{2}(5 \mathrm{~mL})$ was added to the solution dropwise. The resulting mixture was warmed to room temperature and then stirred for another $4 \mathrm{~h}$, after which water $(20 \mathrm{~mL})$ was added, and the organic layer was separated, washed with brine $(30 \mathrm{~mL})$, and dried over anhydrous $\mathrm{MgSO}_{4}$. It was then filtered and concentrated to give a residue, which was purified over silica gel (petroleum/ethyl acetate $=1 / 1$ ) to yield compound $\mathbf{5 d}$ as a white solid (0.5 g, yield: 64\%). mp: $139-141{ }^{\circ} \mathrm{C} .{ }^{1} \mathrm{H}-\mathrm{NMR}\left(400 \mathrm{MHz}, \mathrm{CDCl}_{3}\right) \delta: 1.23(3 \mathrm{H}, \mathrm{t}, J=7.6 \mathrm{~Hz})$, $2.55(2 \mathrm{H}, \mathrm{q}, J=7.6 \mathrm{~Hz}), 3.44(3 \mathrm{H}, \mathrm{s}), 3.77(3 \mathrm{H}, \mathrm{s}), 6.72(1 \mathrm{H}, \mathrm{d}, J=8.4 \mathrm{~Hz}), 6.98(1 \mathrm{H}, \mathrm{d}, J=8.4 \mathrm{~Hz})$, $7.06(1 \mathrm{H}, \mathrm{s}), 7.11(1 \mathrm{H}, \mathrm{d}, J=8.8 \mathrm{~Hz}), 7.22(2 \mathrm{H}, \mathrm{d}, J=8.8 \mathrm{~Hz})$. ESI-HRMS $m / z: 348.10021\left(\mathrm{M}+\mathrm{H}^{+}\right)$ (Calcd for $\mathrm{C}_{18} \mathrm{H}_{19} \mathrm{ClNO}_{4}$ : 348.10026).

\subsection{Biological Activity Test Procedures}

Vero cells were purchased from the American Type Culture Collection and cultured in Minimum Essential Medium (MEM) supplemented with 10\% Fetal Bovine Serum (FBS) and antibiotics (100 U/mL penicillin G, $100 \mu \mathrm{g} / \mathrm{mL}$ streptomycin). Enterovirus 71 (EV 71) strain SZ-98 was kindly provided by Dr. Qi Jin, Institute of Pathogen Biology, Chinese Academy of Medical Science and 
Peking Union Medical School. Enterovirus 71 (EV 71) strain BrCr (VR-1775) was purchased from the American Type Culture Collection. Enterovirus 71 (EV 71) strain JS-52-3 was kindly provided by Dr. Xiang-zhong Ye, Beijing Wantai Biological Pharmacy Enterprise Co., Ltd. All Enterovirus 71 strains were propagated in Vero cells.

All compounds were dissolved in DMSO at $20 \mathrm{mg} / \mathrm{mL}$ as a stock solution and further diluted in culture medium prior to use. The initial concentration in all cytotoxicity assay was $200 \mu \mathrm{g} / \mathrm{mL}$, and inhibition assay for anti-EV71 experiments was from maximum non-toxic concentration, and proceeded serially 3-fold dilution with culture medium. All cytotoxicity and activity assay for each compound was repeated for three times, and the data was indicated as average \pm SD.

\subsubsection{Cytotoxicity Determination}

The cytotoxicity of compounds in the presence of Vero cells was monitored by cytopathic effect (CPE). Vero cells $\left(2.5 \times 10^{4} /\right.$ well $)$ were plated into a 96 -well plate. A total of $24 \mathrm{~h}$ later, the monolayer cells were incubated in the presence of various concentrations of test compounds. After $48 \mathrm{~h}$ of culture at $37{ }^{\circ} \mathrm{C}$ and $5 \% \mathrm{CO}_{2}$ in a carbon-dioxide incubator, the cells were monitored by CPE. The median toxic concentration $\left(\mathrm{TC}_{50}\right)$ was calculated by Reed \& Muench analyses.

\subsubsection{Anti EV 71 Activity Assay}

Confluent Vero cells grown in 96-well microplates were infected respectively with 100 median tissue culture infective dose $\left(\mathrm{TCID}_{50}\right) \mathrm{EV} 71$. After $1 \mathrm{~h}$ adsorption at $37{ }^{\circ} \mathrm{C}$, the monolayers were washed by PBS and incubated at $37{ }^{\circ} \mathrm{C}$ in the maintenance medium with or without different concentrations of test compounds. Viral cytopathic effect (CPE) was observed when the viral control group reached 4 and the antiviral activity of tested compounds was determined by the Reed \& Muench analyses.

\section{Conclusions}

In summary, a series of $N$-phenylbenzamide derivatives were synthesized and their anti-EV 71 activities were evaluated in vitro. All of the synthesized compounds were less toxic to Vero cells in comparison to pirodavir, and several compounds were comparable or superior to pirodavir in terms of SI values, especially compound 1e. This compound has a molecular weight and an ALogP of $321.169 \mathrm{~g} / \mathrm{mol}$ and 2.6 (data obtained from Discovery Studio 3.0), respectively, and shows potential for optimization to improve its activity against EV 71 without sacrificing its druggability. Consequently, compound 1e is a promising lead compound for the development of a new anti-EV 71 agent, and further lead optimization based on compound $1 \mathrm{e}$ will be performed.

\section{Acknowledgments}

This work was supported by the National Natural Science Foundation of China (Grant 30873138 and 81202414). 


\section{References}

1. Schmidt, N.J.; Lennette, E.H.; Ho, H.H. An apparently new enterovirus isolated from patients with disease of the central nervous system. J. Infect. Dis. 1974, 129, 304-309.

2. Samuda, G.M.; Chang, W.K.; Yeung, C.Y.; Tang, P.S. Monoplegia caused by Enterovirus 71: An outbreak in Hong Kong. Pediatr. Infect. Dis. J. 1987, 6, 206-208.

3. Singh, S.; Chow, V.T.; Phoon, M.C.; Chan, K.P.; Poh, C.L. Direct detection of enterovirus 71 (EV71) in clinical specimens from a hand, foot, and mouth disease outbreak in Singapore by reverse transcription-PCR with universal enterovirus and EV71-specific primers. J. Clin. Microbiol. 2002, 40, 2823-2827.

4. Yang, F.; Ren, L.; Xiong, Z.; Li, J.; Xiao, Y.; Zhao, R.; He, Y.; Bu, G.; Zhou, S.; Wang, J.; et al. Enterovirus 71 outbreak in the People's Republic of China in 2008. J. Clin. Microbiol. 2009, 47, 2351-2352.

5. Lee, T.C.; Guo, H.R. Jenny Su, H.J.; Yang, Y.C.; Chang, S.L.; Chen, K.T. Diseases caused by enterovirus 71 infection. Pediatr. Infect. Dis. J. 2009, 28, 904-910.

6. Brown, B.A.; Oberste, M.S.; Alexander, J.P, Jr.; Kennett, M.L.; Pallansch, M.A. Molecular epidemiology and evolution of enterovirus 71 strains isolated from 1970 to 1998. J. Virol. 1999, 73, 9969-9975.

7. Shin, S.R.; Ho, M.S.; Lin, K.H.; Wu, S.L.; Chen, Y.T.; Wu, C.N.; Lin T.Y., Chang L.Y.; Tsao K.C.; Ning H.C.; et al. Genetic analysis of enterovirus 71 isolated from fatal and non-fatal cases of hand, Foot and mouth disease during an epidemic in Taiwan, 1998. Virus Res. 2000, 68, 127-136.

8. Hao, L.H.; Li, Y.P.; He, W.Y.; Wang, H.Q.; Shang, G.Z.; Jiang, J.D.; Li, Y.H.; Li, Z.R. Synthesis and antiviral activity of substituted bisaryl amide compounds as novel influenza virus inhibitors. Eur. J. Med. Chem. 2012, 55, 117-124.

9. Humphrey, J.M.; Chamberlin, A.R. Chemical synthesis of natural product peptides: Coupling methods for the incorporation of noncoded amino acids into peptides. Chem. Rev. 1997, 97, 2243-2266.

10. Huang, Y.P.; Lin, T.L.; Kuo, C.Y.; Lin, M.W.; Yao, C.Y.; Liao, H.W.; Hsu, L.C.; Yang, C.F.; Yang, J.Y.; Chen, P.J.; et al. The circulation of subgenogroups B5 and C5 of enterovirus 71 in Taiwan from 2006 to 2007. Virus. Res. 2008, 137, 206-212.

11. Tan, X.J.; Huang, X.J.; Zhu, S.L.; Chen, H.; Yu, Q.L.; Wang, H.Y.; Huo, X.X.; Zhou, J.H.; Wu, Y.; Yan, D.M.; et al. The persistent circulation of enterovirus 71 in People's Republic of China: causing emerging nationwide epidemics since 2008. PLoS One 2008. 6, e25662.

12. Andries, K.; Dewindt, B.; Snoeks, J.; Willebrords, R.; van Eemeren, K.; Stokbroekx, R.; Janssen, P.A. In vitro activity of pirodavir (R 77975), a substituted phenoxy-pyridazinamine with broad-spectrum antipicornaviral activity. Antimicrob. Agents Chemother. 1992, 36, 100-107.

Sample Availability: Samples of the compounds 1a, 1c, 1e, $\mathbf{2 a}-\mathbf{c}, \mathbf{3 a}-\mathbf{g}$, and $\mathbf{5 a}-\mathbf{f}$ are available from the authors.

(C) 2013 by the authors; licensee MDPI, Basel, Switzerland. This article is an open access article distributed under the terms and conditions of the Creative Commons Attribution license (http://creativecommons.org/licenses/by/3.0/). 